\title{
13. \\ Ueber die Zusammensetzung unendlich kleiner Drehungen.
}

(Yom Hrn. Prof, A. F. Möbius zu Leipzig.)

Der vorliegende Aufsatz über die Zusammensetzung unendlich kleiner Dre• hungen ist eine weitere Ausführung Dessen, was ich in dem unlängst von mir herausgegebenen Lehrbuche der Statik (\$. 183) über diesen Gegenstand gesagt habe. Es wird daselbst zuerst auf die bisher gewöhnliche analytische Art der schon seit lange bekannte Satz*) bewiesen, dafs unendlich kleine Drehungen um Axen, welche sich in einem Puncte schneiden, nach demselben Gesetz, wie Kräfte, zu einer mittleren Drehung sich vereinigen lassen. Hieraus wird mit leichter Mühe weiter geschlossen, dạs auch in allen übrigen Fällen die Zusammensetzung und Zerlegung der Drehungen ganz auf dieselbe Art wie bei Kräften bewerkstelligt werden kạn, Zuletzt wird noch auf die durchgreifende reciproke Beziehung zwischen Kräften und Drehungen aufmerksam gemacht und gezeigt, wie auch die Lehre von den statischen Momenten der Kräfte und damit alle die bisher gefundenen merkwürdigen Sätze dieser Theorie auf Drehungen vollkommene Anweadung finden,

Die hier gegebene Darstellung dieser Gegenstạnde unterscheidet sich von der dortigen, aufser durch gröfsere Ausführlichkeit, hinzugefügte Beispiele und einige daraus gefolgerte geometrische Sätze, hauptsäcklich dadurch, dafs, während ich dort analytisch zu Werke ging, ich mich hier der Construction bedient habe. Es veraṇlafste mich hierzu die Erwägung, dals für einen Gegenstand, der ganz in das Gebiet der Geometrie, und noch dazu des elementaren Theils derselben gehört, eine reine geometrische Behandlung die zweckmäfsigste ist. Ist es mir auf diesem Wege gelungen, den Gegenstand einfạcher als sonst zu entwickeln, so rührt dies besonders noch daher, dafs ich die Theorio der Drehungen mit der Be-

*) Nach Ide (System der reinen und angew, Mechanik fester Körper; Yorrede, Seite xIr.) schreibt sich die Erfiudung dieses Satzes aus Italiè hér. -

Crelle's Journal d. M. Bd. XVIIL. Hft. 3. 
trachtung zweier einander gleichen aber entgegengesetzten Drehungen um parallele Axen eingeleitet habe - eben so, wie auch die Lehre von der Zusammensetzung der Kräfte durch Vorausschickung der Theorie der sogenannten Kräftepaare in hohem Grade vereinfacht wird. *)

§. 1.

Die einfachsten Bewegungen, welche ein Körper annehmen kann, und aus denen alle übrigen sich als zusammengesetzt betrachten lassen, sind die drehende Bewegung um eine in Rube bleibende Axe und das parallele Fortrücken, oder auch nur die drehende Bewegung allein, da man das parallele Fortrücken als eine Drehung um eine unendlich entfernte Axe ansehen kann. Sind demnach zwei verschiedene Lagen eines Körpers gegeben, so mufs es immer möglich sein, ihn durch Drehungen um gewisse Axen aus der einen Lage in die andere zu bringen.

Es läfst sich nun leicht zeigen, dafs zwei Drehungen hierzu immer hinreichend sind. Seien nämlich $A, B, C$ (Fig. 15.) irgend drei, nicht in einer Geraden liegende Puncte des Körpers in der einen Lage und $A^{\prime}, B^{\prime}$, $C^{\prime}$, die Oerter dieser Puncte bei der andern Lage des Körpers, also $\boldsymbol{A B C}$ und $A^{\prime} B^{\prime} C^{\prime}$ zwei einander gleiche und ähnliche Dreiecke. Da die Lage eines Körpers durch die Oerter vollkommen bestimmt ist, welche drei seiner Puncte einnehmen, die nicht in einer Geraden liegen, so wird der Körper aus der einen Lage in die andere gebracht sein, wenn man das eine der beiden Dreiecke $\boldsymbol{A B C}$ und $\boldsymbol{A}^{\prime} \boldsymbol{B}^{\prime} \boldsymbol{C}^{\prime}$ mit dem andern zur Drehung gebracht hat. Man halbire zu $\operatorname{dem}$ Ende die Linie $A A^{\prime}$ durch eine sie normal schneidende Ebene $a$, und man wird $\boldsymbol{A}$ mit $\boldsymbol{A}^{\prime}$ durch Drehung des Körpers um irgend eine in $a$ enthaltene Axe zur Coïncidenz bringen können. Eben so wird $\boldsymbol{B}$ mit $\boldsymbol{B}^{\prime}$ zusammentreffen, wenn man den Körper um eine beliebige Axe dreht, welche in der die Linie $B B^{\prime}$ rechtwinklig halbirenden Ebene $b$ begriffen ist. Dreht man folglich den Körper um die gemeinschaftliche Durchschnittslinie $(a b)$ der Ebenen $a$ und $b$, als Axe, so wird sowohl $A$ nach $A^{\prime}$, als $B$ nach $B^{\prime}$ kommen, und zwar gleichzeitig.

Um das gleichzeitige Zusammentreffen darzuthun, nehme man in der Linie $(a b)$ beliebig zwei Puncte $M$ und $N$ an. Da $M, N$ Punote

*) Vergl. den VII. Band. dieses Journals, Seile 205. 
der Ebene $a$ sind, so ist $M A=M A^{\prime}, N A=N A^{\prime}$, und da $M, N$ atgleich Puncte der Ebene $b$ sind, so ist $M B=M B^{\prime}, N B=N B^{\prime}$. Da nun auch $A B=A^{\prime} B^{\prime}$, so sind die zwei Pyramiden $M N A B$ und $M N A^{\prime} B^{\prime}$ einander gleich und ähnlich und können folglich durch Drehung der einen um die gemeinschaftliche Kante $M N$, d. i. um $(a b)$, zur Cö̈ncidenz gebracht worden, wobei, wie zu beweisen verlangt wurde, $\boldsymbol{A B}$ und $\boldsymbol{A}^{\prime} \boldsymbol{B}^{\prime}$ zusammenfallen.

Um endlich noch $C$ auf $C^{\prime}$ zu bringen, hat man nur den Körper um die jetzt zusammenfallenden $A B$ und $A^{\prime} B^{\prime}$, als $A x e$, zu drehen.

\section{§. 2.}

Zusätze. a) Ein specieller Fall, bei welchem der erste Theil der oben beschriebenen Operation nicht anwendbar ist, ist der, wenn die Ebene $b$ mit der Ebene $a$ zusammenfällt. Alsdann sind $\boldsymbol{A}^{\prime}$ und $\boldsymbol{B} B^{\prime}$ auf derselben Ebene $a$ perpendiculair, folglich diese Linien sowohl, als die Linien $A B$ und $A^{\prime} B^{\prime}$ in einer einzigen Ebene enthalten; der Durchschnitt von $A B$ mit $A^{\prime} B^{\prime}$, welcher $D$ (Fig. 16.) heilse, fält in die Ebene $a$, und es ist $D A=D A^{\prime}$, so wie $D B=D B^{\prime}$. Hier hat man also, um $A B$ auf $A^{\prime} B^{\prime}$ zu bringen, den Körper um eine in $D$ auf der Ebene $A B A^{\prime} B^{\prime}$ normal errichtete Axe zu drehen. Die Coïncidenz von $C$ mit $C^{\prime}$ wird bierauf wie vorhin bewerkstelligt.

b) Nimmt man in dem oben besprochenen Falle, wo die Ebenen $a$ und $b$ eine und dieselben sind, in dieser Ebene irgendwo zwei Puncte $M$ und $N$, welche mit $D$ nicht in einer Geraden liegen, so sind $M N A B$ und $M N A^{\prime} B^{\prime}$ zwei einander gleiche und ähnliche und zugleich symmetrisch liegende Pyramiden. (Liegen die Puncte $M$ und $N$ der Ebene $a$ mit $D$ in einer Geraden, so sind $M N A B$ und $M N A^{\prime} B^{\prime}$ nur ebene Figuren.) Und umgekehrt: baben zwei einander gleiche und ähnliche Pyramiden $M N A B$ und $M N A^{\prime} B^{\prime}$ eine symmetrische Lage, so wird eine Ebene, welche die Linie $A A^{\prime}$ rechtwinklig halbirt, auch $B B^{\prime}$ rechtwinklig halbiren und die gemeinschaftliche Kante $M N$ in sich enthalten. Wenn folglich die Ebene, welche $\boldsymbol{A A ^ { \prime }}$ rechtwinklig halbirt, dieses nicht auch an $B^{\prime}$ thut, d. h. wenn die Ebenen $a$ und $b$ nicht zusammenfallen, so ist es nicht möglich, eine Linie $M N$ so zu ziehen, dafs zwei einander gleiche und äbnliche und zugleich symmetrische Pyramiden $M N A B$ und $M N A^{\prime} B^{\prime}$ entstehen. 
192 13. A. E. Möbius, über die Zusammensetzung unendlich kleiner Drehungen.

Es soll diese Bemerkung zur Ergänzung des in §. 1. geführten Beweises dienen. Unter der dort stillschweigend gemachten Voraussetzung, dafs die Ebenen $a$ und $b$ von einander verschieden sind, können nämlich, dem oben Gesagten zufolge, die einander gleichen und ähnlichen Pyramiden $M N A B$ und $M N A^{\prime} B^{\prime}$ nicht zugleich symmetrisch sein. Sind sie aber dieses nicht, so ist es immer möglich, die eine durch Drehung um $M N$ mit der andern zur Coïncidenz zu bringen. Denn nur in dem Falle, wenn sie zugleich eine symmetrische Lage gegen einander haben, ist eine Coïncidenz auf keine Weise zu bewerkstelligen.

c) Da die drei Puncte $\boldsymbol{A}, \boldsymbol{B}, \boldsymbol{C}$ ganz willkührlich in dem Körper genommen werden können, wofern sie nur nicht in einer Geraden liegen, und da mit anderer Annahme von $A, B, C$ und den ihnen entsprechenden $A^{\prime}, B^{\prime}, C^{\prime}$, in der zweiten Lage des Körpers, auch die Linie $M N$ und die nach der ersten Drehung zusammenfallenden Linien $A B$ und $A^{\prime} B^{\prime}$ ihre Lagen ändern, so erhellet, dafs der Körper auf unendlich viele verschiedene Arten durch zwei Drehungen aus der einen Lage in die andere gebracht werden kann.

d) Zuweilen reicht schon eine einzige Drehung hin, um den Körper aus der einen der beiden gegebenen Lagen in die andere zu bringen. Dieser Fall kann jedoch nur dann eintreten, wenn die drei Linien $A A^{\prime}$, $\boldsymbol{B B}^{\prime}, \boldsymbol{C} \boldsymbol{C}^{\prime}$ einer und derselben Ebene parallel sind. Denn bei einer einzigen Drehung sind die Wege von $A, B, C$ Kreisbögen, deren Ebenen auf der Drehungs - Axe normal und daher mit einander parallel sind. Wenn folglich durch eine einzige Drehung die Puncte $A, B, C$ nach $A^{\prime}, B^{\prime}, C^{\prime}$ kommen, so sind die Linien $A A^{\prime}, B B^{\prime}, C C^{\prime}$, als die Sehnen jener Kreisbögen, einer auf der Drehungs-Axe normalen Ebene parallel.

Wir wollen nun untersuchen, ob auch umgekehrt, wenn $A^{\prime}, B^{\prime}$, $C C^{\prime}$ einer und derselben Ebene $e$ parallel sind, die Dreiecke $A B C$ und $A^{\prime} B^{\prime} C^{\prime}$ durch eine einzige Drehung zur Coïncidenz gebracht werden können. Seien zu diesem Ende $\mathfrak{A}, \mathfrak{B}, \mathfrak{S}, \mathfrak{A}^{\prime}, \mathfrak{B}^{\prime}, \mathfrak{C}^{\prime}$ (Fig. 17.) die rechtwinkligen Projectionen von $A, B, C, A^{\prime}, B^{\prime}, C^{\prime}$ auf $e$, so ist, weil $\boldsymbol{A A}^{\prime}$ mit $e$ parallel ist, $A \mathfrak{A}=A^{\prime} \mathfrak{A}^{\prime}$, und eben so $B \mathfrak{B}=\boldsymbol{B}^{\prime} \mathfrak{B}^{\prime}$. Hieraus und wegen der rechten Winkel bei $\mathfrak{A}, \mathfrak{H}^{\prime}, \mathfrak{B}, \mathfrak{B}^{\prime}$, und da $A B=A^{\prime} B^{\prime}$ ist, folgt weiter, dals die Vierecke $A \mathfrak{A B B}$ und $A^{\prime} \mathfrak{A}^{\prime} \mathfrak{B}^{\prime} B^{\prime}$ einander gleich und ähnlich sind, und dals mithin $\mathfrak{A} \mathfrak{B}=\mathfrak{A}^{\prime} \mathfrak{B}^{\prime}$. ist. Auf gleiche Art zeigt sich, dafs $\mathfrak{B} \mathfrak{C}=\mathfrak{B}^{\prime} \mathfrak{S}^{\prime}$ und $\mathfrak{C} \mathfrak{A}=\mathfrak{E}^{\prime} \mathfrak{A}^{\prime}$ ist. Es sind folglich die Dreiecke 
$\mathfrak{A} \mathfrak{B} \mathfrak{C}$ und $\mathfrak{A}^{\prime} \mathfrak{B}^{\prime} \mathfrak{C}^{\prime}$ in der Ebene $e$ einander gleich und ähnlich, und die Ordnung, in welcher bei ihnen die gleichnamigen Ecken aufeinander folgen, ist entweder eine und dieselbe, oder nicht.

Im ersten Falle liafst sich das eine Dreieck durch Drehung um einen Punct $\mathfrak{M}$ in der Ebene $e$ mit dem andern zur Deckung bringen. Dieser Punct $\mathfrak{M}$ wird gefunden als der gemeinschaftliche Durchschnitt der zwei in $e$ gezogenen, die Linien $\mathfrak{A} \mathfrak{A}^{\prime}$ und $\mathfrak{B} \mathfrak{B}^{\prime}$ rechtwinklig halbirenden Geraden $\mathfrak{a}$ und $\mathfrak{b}$, und, wenn $\mathfrak{a}$ und $\mathfrak{b}$ zusammenfallen', als der gemeinschaftliche Durchschnitt von $\mathfrak{A} \mathfrak{B}$ und $\mathfrak{A}^{\prime} \mathfrak{B}^{\prime}$ selbst. - Der Beweis hiervon wird auf analoge Art, wie vorhin bei den Körpern, geführt. In demselben Falle wird folglich auch der eine Körper mit dem andern durch eine Drehung um eine die Ebene $e$ in $\mathbb{M}$ rechtwinklig schneidende Axe $\mathfrak{m}$ zur Deckung gebracht. Denn hierdurch kommen nicht allein $\mathfrak{A}$, $\mathfrak{B}$, $\mathfrak{C}$ gleichzeitig nach $\mathfrak{U}^{\prime}, \mathfrak{B}^{\prime}, \mathfrak{S}^{\prime}$, sondern auch, da $\mathfrak{A} \boldsymbol{A}$ und $\mathfrak{A}^{\prime} \boldsymbol{A}^{\prime}$ einander gleich und der Drehungs-Axe parallel sind, $A$ gleichzeitig nach $\mathcal{A}^{\prime}$, und eben so $B$ nach $B^{\prime}$ und $C$ nach $C^{\prime}$.

Im anderen Falle, wenn die Aufeinanderfolge der Ecken in dem einen der beiden Dreiecke $\mathfrak{A} \mathfrak{B} \mathfrak{C}$ und $\mathfrak{A}^{\prime} \mathfrak{B}^{\prime} \mathfrak{S}^{\prime}$ der Aufeinanderfolge der gleichnamigen Ecken in dem andern entgegengesetzt ist, wird zwar ebenfalls durch eine Drehung um die Axe $\mathfrak{m}$ die Coïncidenz von $\mathfrak{A} \mathfrak{B}$ mit $\mathfrak{A}^{\prime} \mathfrak{B}$, also auch von $\boldsymbol{A B}$ mit $\boldsymbol{A}^{\prime} \boldsymbol{B}^{\prime}$, aber nicht von $\mathfrak{E}$ mit $\mathfrak{S}^{\prime}$ und daher auch nicht von $C$ mit $C^{\prime}$, herbeigeführt. Da nun, wenigstens im Allgemeinen, die Cö̈ncidenz von $\mathfrak{A} \mathfrak{B}$ mit $\mathfrak{P}^{\prime} \mathfrak{B}^{\prime}$ auf keine andere Weise als durch eine Drehung um die Axe $m$ bewerkstelligt werden kann, so ersieht man, dafs in diesem Falle im Allgemeinen zwei Drehungen nöthig sind, um den Körper aus der einen der beiden gegebenen Lagen in die andere zu bringen.

Nur dann reicht in diesem Falle eine einzige Drehung hin, wenn die Geraden $\mathfrak{a}$ und $\mathfrak{b}$ zusammenfallen, also wenn die Dreiecke $\mathfrak{A} \mathfrak{B} \mathfrak{E}$ und $\mathfrak{A}^{\prime} \mathfrak{B}^{\prime} \mathfrak{C}^{\prime}$ symmetrisch liegen. Alsdann sind $\mathfrak{A} \mathfrak{A}^{\prime}, \mathfrak{B} \mathfrak{B}^{\prime}, \mathfrak{C} \mathfrak{S}^{\prime}$, folglich auch $\boldsymbol{A} A^{\prime}, B B^{\prime}, C C^{\prime}$ miteinander parallel : nicht blofs mit einer und derselben Ebene; und gegen die Ebene, in welcher die Mittelpuncte der drei letzten Linien liegen, haben die Dreiecke $A B C$ und $A^{\prime} B^{\prime} C^{\prime}$ eine symmetrische Lage. Das eine dieser Dreiecke aber wird mit dem andern durch Drehung um die Durchschnittslinie ihrer Ebene zur Coïncidenzgebracht. 
194 13. A. E. IMöbius, über die Zusammensetzung unendlich kleiner Drehungen.

$$
\text { §. } 3 .
$$

Denken wir uns jetzt, dafs ein Körper durch Drehungen um drei oder mehrere verschiedene Axen aus seiner anfänglichen Lage in eine beliebige andere gebracht werde. Da, wie oben gezeigt worden, jede Ortsveränderung eines Körpers, wo nicht durch eine, doch immer durch zwei Drehungen bewerkstelligt werden kann, so werden jene drei oder mehrere Drehungen, wo nicht mit einer, doch immer mit zweien gleichgeltend sein, sich auf zwei reduciren lassen, und es entsteht hiermit die Aufgabe: Aus den Richtungen dreier oder mehrerer Axen und aus den Winkeln, um welche ein Körper um sie gedrelıt wird, swei Axen und die ihnen zugehörigen Drehungswinkel zu finden, dergestalt, da/s die durch letztere Drehungen bewirkte Aenderung der Lage des Körpers einerlei mit der durch erstere Drehungen hervorgebrachten Lage-Aenderung ist.

Die Lösung dieser Aufgabe in ihrer völligen Allgemeinheit möchte zu einem ziemlich verwickelten Resultate führen. Wir wollen uns daher gegenwärtig auf den möglichst einfachen Fall beschränken, in welchem die Drehungswinkel unendlich klein sind, und wo es eben deshalb nicht auf ihre absoluten Werthe, sondern nur auf ihre gegenseitigen Verhältnisse ankommt.

\section{§. 4.}

Bei jeder Drehung kann man seinem eigenen Körper eine solche Lage geben, dafs die Drehung nach einer und derselben Richtung, etwa von der Linken nach der Rechten gebend, erscheint. Man nenne hiernach die positive Richtung ihrer Axe diejenige, bei welcher, wenn mit ihr die Richtung von den Fülsen nach dem Kopfe zusammenfällt, die Drehung nach der Rechten geht. Die Gröfse des unendlich kleinen Drehungswinkels drücke man durch einen ihm proportionalen Theil der Axe aus und verstehe hiernach unter der Drehung $A B$ eine der Länge $A B$ proportionale Drehung um eine Axe, deren positive Richtung vom Puncte $\boldsymbol{A}$ nach dem Puncte $\boldsymbol{B}$ geht. Und eben so, wenn es ohne weiteren $Z_{u}$ satz heifst, dafs der Körper um $\boldsymbol{A B}$ gedreht werden soll, werde durch $A B$ die Richtung der Axe und die Grölse der Drehung zugleich ausgedrückt angenommen.

Sind daher $A, B, C, D, \ldots$ mehrere in einer Geraden liegende Puncte, so sind die Drehungen $\boldsymbol{A B}$ und $\boldsymbol{B C}$ gleichwirkend mit der ein- 
zigen $A C$, die Drehungen $A B, B C$ und $C D$ gleichwirkend mit der einzigen $A D$, u. s. w., in welcher Ordnung auch die Puncte aufeinander folgen mögen.

$$
\text { §. } 5 \text {. }
$$

Bei der Drehung $\boldsymbol{A B}$ (Fig. 18.) beschreibt der Punct $\boldsymbol{P}$ des Körpers eine unendlich kleine, auf der Ebene $A B P$ perpendiculaire Linie, welche proportional mit der Grülse der Drehung, d. i. mit $\boldsymbol{A B}$, und mit der Entfernung des Punctes $P$ von $\boldsymbol{A B}$, also proportional mit der Dreiecksfläche $\boldsymbol{P} A B$ ist. Die Richtung aber, nach welcher $\operatorname{sich} \boldsymbol{P}$ bewegt, ist einerlei mit der positiven Richtung einer Axe, welche einer, ihrem Sinne nach, durch die Aufeinanderfolge $\boldsymbol{P A B}$ angedeuteten Drehung zukommt. Ist nämlich $\boldsymbol{P P}^{\prime}$ der von $\boldsymbol{P}$ beschriebene Weg, und erscheint dieser, wenn $\boldsymbol{A B}$ die Richtung von den Füfsen nach dem Kopfe ist, nach rechts gehend, so wird auch, wenn $\boldsymbol{P P}^{\prime}$ zur Richtung von den Füfsen nach dem Kopfe genommen wird, die Richtung $\boldsymbol{A B}$, folglich auch der Sinn der Drehung $\boldsymbol{P} \boldsymbol{A B}$, nach rechts gehen.

Bei mehreren Drehungen $A B, C D, \ldots$ um Axen, welche in einer und derselben Ebene liegen, sind daher die Bewegungen eines in derselben Ebene befindlichen Punctes $P$ nicht blofs der Grölse, sondern auch dem Zeichen nach, den Dreiecken $P A B, P C D, \ldots$ proportional, indem die bei den Drehungen $A B$ und $C D$ auf der Ebene normalen Wege von $\boldsymbol{P}$ nach einerlei oder entgegengesetzten Seiten dieser Ebene gerichtet sind, je nachdem die Folgen $P A B$ und $P C D$ von einerlei oder entgegengesetztem Sinne sind, also die Dreiecke $P A C$ und $P C D$ einerlei oder verschiedene Zeichen haben.

§. 6.

Seien $\boldsymbol{A}, \boldsymbol{B}, \boldsymbol{C}, \boldsymbol{D}$ (Fig. 19.) die vier aufeinander folgenden Ecken eines Parallelogramms, und $C^{\prime}, D^{\prime}$ die Oerter, welche die anfänglich mit $C, D$ zusammenfallenden Puncte des Körpers nach einer Drehung $A B$ einnehmen. Eben so seien $A^{\prime}, \boldsymbol{B}^{\prime}$ die Oerter der anfänglich mit $\boldsymbol{A}, \boldsymbol{B}$ zusammenfallenden Puncte des Körpers nach einer Drehung $C D$. Da die Puncte $C^{\prime}, D^{\prime}$ resp. den $C, D$ unendlich nahe liegen, so bleiben sie bei der zweiten Drehung $\boldsymbol{C D}$ unverrückt, und die nach beiden Drehungen von $A, B, C, D$ beschriebenen Wege $A A^{\prime}, B B^{\prime}, C C^{\prime}, D D^{\prime}$ sind auf der Ebene $A B C D$ rechtwinklig und resp. proportional den Dreiecken $A C D$, $B C D, C A B, D A B$, also einander gleich: auch dem Zeichen nach; so dafs 
durch beide Drehungen ein paralleles Fortrïcken des Körpers ohne Drehung um eine der Hälfte des Parallelogramms $A B C D$ proportionale Linie bewirkt wird.

Ueberhaupt also erzeugen zwei einander gleiche aber entgegengesetzte Drehungen um zwei parallele Axen ein auf der Ebene der Axen perpendiculares Fortrücken, welches dem Producte aus dem gegenseitigen Abstande der beiden Axen in den Drehungswinkel proportional ist.

$$
\text { §. } \% \text {. }
$$

Seien $\boldsymbol{A}, \boldsymbol{B}, \boldsymbol{C}$ drei nicht in einer Geraden liegende Puncte, und werde der Körper nach und nach um $A B, B C, C A$ gedreht. Ein in der Ebene $A B C$ befindlicher Punct $P$ des Körpers rückt dabei rechtwinklig auf der Ebene fort, um Linien, die den Dreiecken $P A B, P B C, P C A$ proportional sind. Die Totalverrückung von $P$ ist daher der Summe dieser Dreiecke, mit gehöriger Berücksichtigung ihrer Zeichen, proportional. Diese Summe ist aber gleich dem Dreiecke $A B C$, also unabhängig von dem Orte des Punctes $P$ in der Ebene $A B C$. Mithin rückt jeder Punct dieser Ebene, und folglich der Körper selbst, perpendioular auf derselben, um eine der Dreiecksfläche $\boldsymbol{A B C}$ proportionale Gröfse fort.

Zusatz. Auf gleiche Art wird bewiesen, dafs auch bei einem mehrseitigen ebenen Vieleck, wenn jede seiner Seiten nach der Richtung genommen wird, nach welcher sie von einem den Perimeter des Vielecks beschreibenden Punct durchlaufen wird, der um jede Seite um einen ihrer Länge proportionalen Winkel gedrehte Körper perpendicular auf der Ebene des Vielecks um eine der Fläche des letzteren proportionale Linie fortrückt.

Dieses parallele Fortrücken des Körpers findet selbst dann Statt, wenn das Vieleck kein ebenes ist. Denn sind z. B. $A, B, C, D$ vier nicht in einer Ebene liegende Puncte, so bewirken die Drehungen $\boldsymbol{A B}$, $B C, C A$ ein auf der Ebene $A B C$ perpendiculares Fortrioken, und die Drehungen $A C, C D, D A$ ein auf der Ebene $A C D$ perpendiculares Fortrücken. Von der einen Seite reduciren sich aber diese 6 Drehungen auf die vier $A B, B C, C D, D A$, indem sich $C A$ und $A C$ gegen einander aufbeben, und von der andern erzeugen zwei verschieden gerichtete parallele Fortrückungen in Verbindung ein paralleles Fortrícken nach einer mittleren Richtung. 


\section{8.}

Seien wiederum $\boldsymbol{A}, \boldsymbol{B}, \boldsymbol{C}, \boldsymbol{D}$ die vier Ecken eines Parallelogramms, in ihrer Aufeinanderfolge, so sind die zwei Drehungen $A B, C D$ gleichwirkend mit den dreien $\boldsymbol{A B}, \boldsymbol{B C}, \boldsymbol{C A}$, indem erstere sowohl (§. 6.), als letztere (\$. 7.), ein dem Inhalte des Dreiecks $A B C$ proportionales und auf seiner Ebene perpendiculares Fortrücken nach derselben Seite hervorbringen. Mithin sind auch, wenn auf beiden Seiten die Drehungen $B A, C B$ hinzugefügt werden, die zwei Drehungen $C B$ und $\dot{C D}$ gleichwirkend mit der einzigen $\boldsymbol{C A}$; d. h. zwei Drehungen, deren Axen sich in einem Puncte schneiden, lassen sich zu einer einzigen zusammensetzen, welche, der Richtung ihrer Axe und ihrer Grö/se nach, durch die Diagonale eines Parallelogramms ausgedrückt voird, in welchem die anliegenden Seiten, iltrer Richtung und Grö/se nach, die erstern Drelungen vorstellen.

Zusatz. Aus der Zusammensetzung zweier Drehungen, deren Axen sich schneiden, mufs sich die Zusammensetzung von Drehungen um parallele Axen, als ein specieller Fall der erstern, herleiten lassen, da parallele Axen auch als solche angesehen werden können, welche sich in unendlicher Entfernung schneiden. Indessen lifst sich dieser besondere Fall auch unmittelbar durch Anwendung des in \$. 6 erhaltenen Satzes behandeln.

Seien $A B C D$ und FGHI Fig. 20. zwei in einer Ebene liegende Parallelogramme, und ihre Flächen auch den Zeichen nach einander gleich, so dafs durch die Folgen $A B C$ und $F G H$ einerlei Sinn der Umdrebung ausgedrückt wird, Alsdann haben die Drehungen $A B$ und $C D$ gleiche Wirkung mit den Drehungen $\boldsymbol{F G}$ und $\boldsymbol{H I}$, da jedes dieser Paare von Drehungen ein auf der Ebene ihrer Axen perpendiculares, nach einerlei Seite gerichtetes und dem Inhalte der Parallelogramme proportionales Fortriicken hervorbringt. Es heben sich daher die vier Drehungen $A B, C D, G F$, IH gegen einander auf. Werden nun noch, was immer möglich, die zwei Parallelogramme in einer solchen Lage gegen einander angenommen, dafs $C D$ und $G F$ in dieselbe Grade fallen und darin eiverlei Richtung haben, so ist diese Gerade parallel mit $A B$ und $I H$ und liegt zwischen ihnen, in Abständen, die sich wegen der Gleichheit der Parallelogramme wie $I H$ zu $A B$ verhalten. Dabei werden die Drehungen $C D$ und $G F$ gleichwirkend mit der einzigen $C D+G F$, und wir schliefsen daher:

Wenn von drei Drehungen um parallele Axen in einer Ebene die Drehung um die mittlere Axe den entgegengesetzten Sinn der beiden anCrelle's Journal d. M. Brl. XViII. Hft. 2. 
108 13. A. E. Möbius, über die Zusammensetzung unendlich kleiner Drehungen.

dern hat und der Summe derselben gleieh ist, und wenn die mittlere Axe von den beiden andern sich in Abständen befindet, die sich umgekehrt wie die Drehungen um dieselben verhalten, so heben sich die Drehungen gegen einander auf.

Wie hiernach Drehungen um zwei parallele Axen zu einer dritten verbunden werden können, ist ohne weiteres Erörtern klar.

$$
\text { §. } 9 .
$$

Wenn in dem Vorhergehenden gezeigt wurde, wie zwei oder mehrere Drehungen ein paralleles Fortrücken hervorbringen, und wie zwei Drehungen sich zu einer einzigen zusammensetzen lassen, so war, wie sich schon aus den dort angewendeten Schlüssen ergab, die Ordnung, in welcher man die zwei oder mehrere Drehungen auf einander folgen liefs, ganz willkührlich. Es läfst sich aber diese Willkühr in der Aufeinanderfolge unendlich kleiner Drehungen, abgesehen von jenen speciellen Fällen, auch allgemein darthun. Sie bildet einen Fundamentalsatz in der Theorie der Drehungen und gründet sich darauf, dafs die Wege, welche zwei einander unendlich nahe Puncte des Körpers bei einer unendlich kleinen Drehung desselben beschreiben, als zwei einander gleiche Parallelen $\mathrm{zu}$ betrachten sind.

Wenn daher der Punct $A$ (Fig. 21.) bei der Drehung $F G$ den Weg $A B$, und bei der Drehung $H I$ den Weg $A D$ beschreibt, so wird er, wenn nach der ersten dieser Drehungen die zweite $\boldsymbol{H}$ erfolgt, von $B$ aus, als von einem Puncte, dessen Lage gegen die Axe $\boldsymbol{H}$ von der Lage des $\boldsymbol{A}$ gegen dieselbe Axe nur unendlich wenig verschieden ist, einen dem $A D$ gleichen und parallelen Weg zurücklegen. Ist er aber zuerst durch die Drehung $H I$ von $A$ nach $D$ gebracht worden, so wird er, wenn hierauf die Drehung $\boldsymbol{F} G$ geschieht, von $D$ aus einen Weg, gleich und parallel mit $\boldsymbol{A B}$, beschreiben. Mag also zuerst die Drehung $\boldsymbol{F G}$ und nachher die Drehung $\boldsymbol{H}$, oder umgekehrt, erfolgen, so wird der Punct $\boldsymbol{A}$ immer nach der vierten Ecke $C$ des Parallelogramms gelangen, dessen drei übrige Ekken in ihrer Folge $\boldsymbol{B}, \boldsymbol{A}, \boldsymbol{D}$ sind. Da nun dasselbe auch von allen übrigen Puncten des Körpers gilt, so schliefsen wir, dafs die durch zwei unendlich kleine Drehungen um zwei verschiedene Axen bewirkte Verrückung unabbängig jist von der Aufeinanderfolge der Drehungen. Da endlich von irgend zwei Complexionen dreier oder mehrerer Elemente die eine aus 
der andern durch fortgesetztes Vertauschen zweier nebeneinanderstehender Elemente hergeleitet werden kann, so erhellet, dafs auch die durch drei oder mehrere Drehungen bewirkte Totalverrückung von der Ordnung, in welcher die Drehungen auf einander folgen, unabhängig ist.

Mit Hülfe dieses Satzes lassen sich auch die im vorigen $\S_{\bullet}$ in Anwendung gekommenen Sätze beweisen, nämlich, dafs wenn eine oder mehrere Drehungen gleiche Wirkung mit mehreren andern Drehungen haben, die erstern, in Verbindung mit den in entgegengesetzten Sinne genommenen letztern, den Körper unverrückt lassen, und dafs, wenn mehrere Drehungen einander aufheben, eine oder etliche derselben gleichwirkend mit den entgegengesetzt genommenen übrigen sind.

Denn sind die Drehungen $A B, C D, \ldots$ gleichwirkend mit den Drehungen $F G, H I, \ldots$, so sind es auch $A B, C D, \ldots G F, I H, \ldots$ mit $\boldsymbol{F G}, \boldsymbol{H I}, \ldots G \boldsymbol{G F}, \boldsymbol{I H}, \ldots$ Letztere aber, in der Ordnung $\boldsymbol{F G}, \boldsymbol{G F}, \boldsymbol{H}$, IH .... genommen, heben sich paarweise auf. Mithin muls auch die Totalwirkung der erstern Null sein.

Auf ähnliche Art wird auch der umgekehrte Satz bewiesen.

\section{§. 10.}

Die Art und Weise, auf welche sich nach \$.4. und \$. 8. zwei oder mehrere Drehungen, deren Axen in dieselbe Gerade fallen, und zwei Drehungen, deren Axen in derselben Ebene liegen, zu einer einzigen zusammensetzen lassen, ist ganz der Zusammensetzung von Kräften analog, deren Richtungen in derselben Geraden oder in derselben Ebene enthalten sind. Man hat nämlich nur Richtung und Grölse der Kraft mit Axe und Gröfse der Drehung zu vertauschen, um aus der bekannten Vorschrift für die Zusammensetzung der Kräfte die Vorschrift für die der Drehungen abzuleiten. Da nun, wie aus der Statik bekannt ist, mit Anwendung des Satzes von Kräften, deren Richtungen in dieselbe Gerade fallen, und mit Hülfe des Parallelogramms der Kräfte, alle übrigen die Zusammensetzung und Zerlegung der Kräfte, betreffenden Sätze sich ergeben, so erhellet, dafs nicht allein in den vorhin besonders betrachteten Fällen, sondern auoh in allen übrigen zwischen Kräften und Drehungen, die vollkommenste Analogie herrschen mufs. Unter andern werden daher alle die merkwürdigen Eigenschaften, welche die sogenannten Kräftepaare besitzen, aueh Paaren von Drébungen, d. i. Paaren yon einander gleichen aber entgegen- 
200 13. A. E. Möbius, über die Zusammensetzung unendlich kleiner Drehungen.

gesetzten Drehungen um parallele Axen zukommen. So folgt z. B. schon unmittelbar aus \$.6., dafs, eben so wie ein Kräftepaar, auch ein Paar von Drehungen ohne Aenderung seiner Wirkung in seiner Ebene und parallel mit derselben verlegt werden kann.

Ueberhaupt also: wenn zwischen mehrern, auf einem frei beweglichen Körper wirkenden Kräften Gleichgewicht statt findet, wird auch der Total-Effect von Drehungen, deren Axen die Richtungen der Kräfte sind, und deren Grö/sen sich wie die Intensitäten der Kräfte verhalten, Null sein; oder kürzer ausgedrückt: Halten die ihrer Richtung und Intensitäit nach durch die Linien $A B, C D, E F, \ldots$ dargestellten Kräfte einander das Gleichgewicht, so bringen auch die Drehungen $A B, C D$, $E F, \ldots$ in Verbindung keine Verrückung hervor.

Insbesondere fliefst hieraus noch, dafs - da drei oder mehrere auf einen Körper wirkende Kräfte sich immer, wo nicht auf eine, doch auf zwei reduciren lassen, - dafs auch Drehungen in beliebiger Anzahl immer auf wenigstens zwei zurückgeführt werden können. Auch fołgerten wir dasselbe schon in $\S .3$. aus den in \$§. 1. und 2. angestellten Betrachtungen.

\section{§. 11.}

Die zwischen Kräften und Drehungen stattfindende Analogie ist noch besonders wegen ihrer reciproken Beschaffenheit merkwürdig. Eine einzelne Kraft wird bestimmt durch die Gerade, in welcher sie wirkt, durch ihre doppelt mögliche Richtung in dieser Geraden und durch ihre Grölse. Die Wirkung zweier einander gleichen, parallelen und entgegengesetzten Kräfte, oder eines Kräftepaares, wird bestimmt durch irgend eine auf der Ebene des Paares perpendiculare Linie, durch den Sinn der Drehung um diese Linie und durch das Moment des Paares oder das Product aus der einen der beiden Kräfte in ihre Entfernung von der andern. Denn jedes andere Paar, bei welchem die genannten Stücke dieselben sind, hat mit ersterem gleiche Wirkung.

Eben so ist nun, indem man fortrückende und drehende Bewegung mit einander vertauscht, eine einzelne Drehung durch ihre Axe, ihren Sinn und ihre Gröfse bestimmt. Ein Paar von Drehungen aber ist es durch irgend eine auf der Ebene der Axen beider Drehungen perpendiculare Linie, durch die Richtung, nach welcher der Körper in dieser Linje fortgerückt wird, und durch die Grölse dieses Fortrückens, welches nach 
§. 6. dem Producte aus der einen der beiden Drehungen in den gegenreitigen Abstand der beiden Axen gleich ist und daber analogerweise das Moment des Paares von Drehungen heifsen kann.

\section{§. 12.}

Auf ähnliche Art läfst sich auch der Begriff des Moments einer Kraft, in Bezug auf eine gewisse Axe, auf Drehungen anwenden. Das Moment einer Kraft, in Bezug auf eine Axe, drückt die Grölse aus, mit welcher die Kraft den Körper, an welchen sie angebracht ist, um die Axe, wenn diese festgehalten wird, zu drehen strebt; denn es herrscht Gleichgewicht, wenn die Momente zweier Kräfte, welche den Körper nach entgegengesetzten Richtungen um eine feste Axe zu drehen streben, in Bezug auf diese Axe einander gleich sind. Indem wir daher das Drehen mit dem Fortrücken vertauschen, wird das auf eine Axe $M N$ bezogene Moment der Drehung $\boldsymbol{A B}$ der Grölse proportional sein, um welche die mit $M N$ anfangs zusammenfallenden Puncte des Körpers längs $M N$ durch die Drehung $A B$ fortgerückt werden. Folgende Betrachtungen werden dieses in noch helleres Licht setzen.

Wenn erstens $M N$ mit $A B$ in einer Ebene liegt, so rückt bei der Drehung um $\boldsymbol{A B}$ jeder Punct dieser Ebene perpendicular auf derselben, und daher auch jeder Punct in $M N$ perpendicular auf $M N$ fort, und es ist folglich das Fortrücken jedes Punctes in $M N$ längs $M N$, also auch das Moment der Drehung $\boldsymbol{A B}$ in Bezug auf $\boldsymbol{M N}$, $=0$, eben so, wie das Moment einer Kraft für jede Axe, die mit der Kraft in einer Ebene liegt, $=0$ ist.

Man nehme ferner an, dafs $A B$ und $M N$ nicht in einer Ebene begriffen und zuerst unter einem rechten Winkel gegen einander geneigt sind. Alsdann ist es möglich, durch $\boldsymbol{A B}$ eine Ebene zu legen, welche von $\boldsymbol{M N}$ rechtwinklig geschnitten wird. Durch den Schneidepunct, welcher $D$ (Fig. 22.) heifse, lege man eine Linie $D C$, gleich und parallel der $A B$, welche mithin in letztgedachter Ebene enthalten sein wird. Nun bewirken die Drehungen $A B$ und $C D$ ein auf der Ebene $A B D$ perpendiculares und daher mit $M N$ paralleles Fortrücken, welches der Fläche $A B D$ proportional ist. Durch diese zwei Drehungen wird folglich jeder in $M N$ fallende Punct, in $M N$ selbst, um eine mit $A B D$ proportionale Grölse fortgerückt. Da aber $M N$ mit $C D$ in einer Ebene liegt und daher die 
Drehung $C D$ zur Bewegung der Puncte in $M N$ lings $M N$ nichts beiträgt, so rückt schon durch die Drehung $A B$ allein jeder in $M N$ fallende Punct längs $M N$ um ein gleich grofses Stück fort, welches mit $\boldsymbol{A B D}$, d. i. mit dem Producte aus der Drehung in den Abstand ihrer Axe von der Momenten-Axe, proportional und als das Moment der Drehung $A B$ in Bezug auf $\boldsymbol{M N}$ auzusehen ist.

Nimmt man den durch $M N$ ausgedrïckten Theil der MomentenAxe von constanter Länge, so kann man das Moment, anstatt dem Dreieck, $A B D$, auch dem Producte $\frac{1}{3} M N . A B D$, d. i. der Pyramide $A B M N$ proportional setzen. Denn, da $A B D$ von $M N$ in $D$ perpendicular getroffen wird, so ist, wie man leicht sieht, die Pyramide $A B M N$ einer andern gleich, welche $\boldsymbol{A B D}$ zur Grundfläche und $M N$ zur Höhe hat.

Wenn zweitens die nicht in einer Ebene enthaltenen $A B$ und $M N$ einen schiefen Winkel mit einander machen, so beschreibe man, um diesen Fall auf den rorigen zurückzuführen, um $A B$, als Diagonale, eis Rechteck $A F B G$ (Fig. 23.), dessen Seite $A G$ mit $M N$ parallel ist, und dessen Seite $\boldsymbol{A F}$ folglich einen rechten Winkel mit $M N$ macht. Hiernach ist die Drehung $A B$ gleichwirkend mit den Drehungen $A G$ und $A F$, Von diesen bringt erstere, da ihre $A x \theta A G$ mit $M N$ in einer Ebene liegt, keine Verrückung der Puncte in $M N$ längs $M N$ hervor. Die durch die Drehung $A B$ erzeugte Verrückung ist folglich einerlei mit der durch die Drehung $A F$ erzeugten, d. h. die Drehungen $\boldsymbol{A B}$ und $\boldsymbol{A F}$ haben in Bezug auf $M N$ einander gleiche Momente. Nach dem Vorigen ist aber das Moment der Drehung $\boldsymbol{A F}$ proportional der Pyramide $\boldsymbol{A F M N \text { , und }}$ diese ist gleich der Pyramide $\boldsymbol{A B M N}$, weil beide eine gemeinschaftliche Grundflïche $A M N$ haben und die Linie durch ihre Spitzen $F$ und $B$, parallel mit $\boldsymbol{A G}$, folglich mit $M N$, folglich auch mit der Grundfläche ist. Das Moment der Drehung $\boldsymbol{A B}$ in Bezug auf $\boldsymbol{M N}$ ist daher, auch bei jeder beliebigen Lage von $M N$ gegen $A B$, der Pyramide $A B M N$ proportional; d. h. es wird durch die Drehung $A B$ jeder in die Linie $M N$ fallende Punct längs $M N$ um ein gleiches Stück fortgerückt; welches Stück, wenn der Abschnitt $M N$ dieser Linie von constanter Länge genommen wird, der Pyramide proportional ist, welche diesen Abschnitt und die die Drehung darstellende Linie $\boldsymbol{A B}$ zu gegenüberstehenden Seiten hat.

Eine unmittelbare Folge hiervon ist, dafs auch die durch mehrere Drehungen $A B, C D, \ldots$ bewirkte Verrückung längs $M N$ für alle Puncte 
13. A. E. Möbius, über die Zusammensetzung unendlich kleiner Drehungen. 203 in $M N$ von gleicher Grọ̈se ist, nämlich von einer Gröfse die der Summe der Pyramiden $\boldsymbol{A B M N}+\boldsymbol{C D} \boldsymbol{M N}+\ldots$, mit gehöriger Rücksicht auf ihre Zeichen, proportional ist und das Moment des Systems der Drehungen $A B$, $C D, \ldots$ in Bezug auf die Axe $M N$ genannt werden kann. Und da nach §. 1. und 2. jede Verrückung eines Körpers durch zwei Drehungen, wenn nicht durch eine einzige, hervorgebracht werden kann, so wird auch durch jede beliebige Verrückung jeder Punct einer beliebig gewählten Axe $M N$ um ein gleich grofses Stück längs $M N$ verrückt werden; und dieses Stück wird als das Moment der Verrückung in Bezug auf $M N$ anzusehen sein.

\section{\$. 13.}

Zwischen Kräften und Drehungen herrscht demnach auch hinsichtlich der Momente eine vollkommene Analogie. Denn, wie sich zeigen lïist *), kann das Moment der Kraft $A B$ in Bezug auf die Axe $M N$ geometrisch durch die Pyramide $\boldsymbol{A B M N}$ dargestellt werden. Alle die merkwürdigen Sätze, welche hinsichtlich der Momente von Kräften gelten, finden folglich auch hier Statt.

Dem Satze z. B., dals, wenn ein System von Kräften im Gleichgewichte ist, die Summe der Momente der Kräfte des Systems, oder kürzer, das Moment des Systems, in Bezug auf jede beliebige Axe Null ist, entspricht folgender Satz. Wenn mehrere Drehungen sich gegenseitig aufheben, so ist ihr Moment, d. $i$. die Summe der Momente der einzelnen Drehungen, in Bezug auf jede beliebig gewählte Axe, Null. Auch folgt die Richtigkeit dieses Satzes schon unmittelbar aus dem vorhin gegebenen Begriff des Moments einer Drehung. Denn wenn ein Körper nach mehrern Drehungen in seine anfängliche Lage zurïckgekommen ist, so hat auch kein Punct desselben längs einer durch den Punct gehenden geraden Linie (Axe des Moments) seine Lage geändert.

Da ferner, wenn die drei Momente mehrerer in einer Ebene wirkender Krifte in Bezug auf drei die Ebene rechtwinklig schneidende und nicht in einer Ebene liegende Axen einzeln Null sind, die Kräfte sich das Gleichgewicht halten, so können auch mehrere Drehungen, deren Axen in einer Ebene liegen, wenn ihre drei Momente für drei auf der Ebene nor-

*) Vergl. einen Anfoatz des Verf. im 4ten Bande dieses Journals, Seite 179, so wie such des Verf. Lehrb. der Statik. $§ .59$. 
mal stehende und nicht in einer Ebene enthaltene Axen einzeln Nuil sind, keine Verrickung hervorbringen. Uebrigens folgt auch dieser Satz ganz leicht aus der von den Momenten aufgestellten Erklïrung. Da nämlich alle Drehungs-Axen in einer und derselben Ebene sein sollen, so mufs die durch die einzelnen Drehungen erzeugte Bewegung der Puncte der Ebene auf diese perpendicular sein; und da die Momente für drei die Ebene perpendicular sohneidende Axen Null sein sollen, so haben die drei Schneidopuncte gar keine Bewegung. Diese drei Puncte liegen aber nicht in gerader Linie, weil die drei Axen nicht in einer Ebene liegen sollen; und wenn drei Puncte eines Körpers, die nicht in einer Geraden liegen, keine Bewegung haben, so ist auch der Körper selbst in Ruhe.

\section{\$. 14.}

Einer der fruchtbarsten Sätze in der statischen Theorie der Momente ist folgender. Bestimmt man die Momente eines System $\mathbf{S}$ von Kräften rücksichtlich mehrerer Axen, und kann man nach der Richtung einer jeden dieser Axen eine Kraft wirken lassen, von der Grölse, dafs alle diese neuen Krïfte einander das Gleichgewicht halten, 80 ist die Summe der Momente von $\boldsymbol{S}$, jedes Moment vorher mit einem Coëfficienten multiplicirt, welcher der der Axe des Moments zugehörigen Kraft proportional ist, $=0 *$ ).

Auf die Theorie der Drehungen angewendet, lautet dieser Satz also: Bestimmt man die Momente einer beliebigen Verrückung eines Körpers in Bezug auf mehrere Axen, und kann man für diese Axen Drehungswinkel finden, die in solchen Verhültnissen zu einander stehen, da/s alle diese Drehungen einander aufheben, so ist die Summe der Momente der Verrückung, jedes Moment vorher mit einem Coëfficienten multiplicirt, welcher dem der Axe des Moments zugehörigen Drehungsuinkel proportional ist, $=0$.

Der Beweis dieses Satzes dürfte sich folgendergestalt am einfachsten geben lassen.' Man bezeichne durch $a, b, c, \ldots$ ihrer Lage und Richtung nach bestimmte Geraden; durch $t, u, v, \ldots$ Zahlen; durch $a_{i}, b_{u}, \ldots$ Abschnitte der Linien $a, b, \ldots$, , welche resp. $t, u, \ldots$ Linien-Einheiten lang sind; durch $\left[a_{t} b_{u}\right]$ endlich den Inhalt einer Pyramide, welohe die Abschnitte

*) Des Verf. Lelurbnch der Statik, §. 99. 
$a_{t}$ und $b_{u}$ zu gegenüberliegenden Seiten hat. Es bedarf kaum der Erinnerung, dafs durch $a, b, t, u$ der Inhalt der Pyramide $\left[a_{t} b_{u}\right]$ vollkommen bestimmt ist, wenn auch in den Linien $a$ und $b$ die Anfangspuncte der Abschnitte $a_{t}$ und $b_{u}$ unbestimmt gelassen werden. Auch ist, wie man leicht sieht : $a_{t}=t . a_{1},\left[a_{t} b_{u}\right]=t\left[a_{1} b_{u}\right]=u\left[a_{t} b_{1}\right]=t u\left[a_{1} b_{1}\right]=\left[a_{u} b_{t}\right]=$ etc. , wo $a_{1}, b_{1}$ eine Linien - Einheit lange Abschnitte der Linien $a, b$ bedeuten.

Sei nun die in Rede stehende Verrückung durch zwei den Zahlen $\boldsymbol{t}, \boldsymbol{u}$ proportionale Drehungen um die Axen $a$ und $b$ erzeugt worden (\$. 12 zu Ende). Sei ferner der Total - Effect der Drehungen $x, y, z, \ldots$ um die Axen $f, g, h, \ldots$ Null, und daher, wenn man das Moment dieses Systems von Drehungen das einemal auf $a$, das anderemal auf $b$ als Axen bezieht und diesen Axen resp. die Längen $t$ und $u$ giebt :

und eben so

$$
\begin{aligned}
& \quad\left[a_{t} f_{x}\right]+\left[a_{t} g_{y}\right]+\left[a_{t} h_{z}\right]+\ldots=0, \text { oder } \\
& x\left[a_{t} f_{1}\right]+y\left[a_{t} g_{1}\right]+z\left[a_{t} h_{1}\right]+\ldots=0,
\end{aligned}
$$

$$
x\left[b_{u} f_{1}\right]+y\left[b_{u} g_{1}\right]+z\left[b_{u} h_{1}\right]+\ldots=0 .
$$

Die Summe dieser zwei Gleichungen ist aber der analytische Ausdruck des zu beweisenden Sạtzes, Es sind nämlich

$$
\left[a_{t} f_{1}\right]+\left[b_{u} f_{1}\right], \quad\left[a_{t} g_{1}\right]+\left[b_{u} g_{1}\right], \quad\left[a_{t} h_{1}\right]+\left[b_{u} h_{1}\right], \text { u. s. w, }
$$

die Momente der durch $a_{t}$ und $b_{u}$ bestimmten Verrückung in Bezug auf die (gleich langen) Axen $f, g, h, \ldots$, , und diese Momente haben in der Summengleichung die Coëfficienten $x, y, z \ldots$.

Beispiel. Sind $f, g, h$ drei sich in einem Puncte $I$ schneidende und in einer Ebene liegende Geraden, so ist damit ein Parallelogramm FGHI seiner Form nach gegeben, dessen Ecken $F, G, H$ resp. in $f, g, h$ liegen; und die Drehungen um $f, g, h$ heben sich auf, wenn sie ihrer Grölse nach mit IF, GI, IH proportional sind. Wenn daher $p, q, r$ die Momente irgend einer Verrückung in Bezug auf $f, g, h$ sind, so ist

so wie

$$
\boldsymbol{I F} \cdot \boldsymbol{p}+\boldsymbol{G I} \cdot q+\boldsymbol{I H} \cdot \boldsymbol{r}=0
$$

$$
I F \cdot p+I H \cdot r=I G \cdot q_{p}
$$

In diesen Gleichungen ist jedes Glied, z. B. IF.p, positiv oder negativ zu nehmen, je nachdem die Richtung $I F$ in $f$ und die Richtung, nach welcher die in $f$ fallenden Puncte längs $f$ um $p$ bei der Verrückuvg sich fortbewegen, einerlei oder entgegengesetzt sind.

Crelle's Journal d. M. Bd. XVILL Hft. 3. 
206 13. A. E. Möbius, über die Zusammensetzung unendlich kleiner Drehungen.

\section{15.}

Wenn für gegebene Axen sich Drehungen finden lassen, welche einander aufheben, so werden auch Kräfte, welche die Richtungen der Axen haben, und den Drebungen proportional sind, das Gleichgewicht sich halten. In $\$$. 98. und 99. des gedachten Lehrbuchs der Statik habe ich unteisucht, welchen Bedingungen die gegenseitige Lage von 2, 3 und mehreren Richtungen unterworfen sein mufs, wenn Kräfte sich sollen angeben lassen, die, nach diesen Richtungen wirkend, im Gleichgewichte sind. Es ergaben sich hierbei, wenn die Anzahl der Richtungen kleiner als 7 war, gewisse positive Bedingungen für ihre gegenseitige Lage. So mufs z. B. bei 4 Richtungen jede Gerade, welche 3 derselben trifft, auch der 4ten begegnen. Für 7 Richtungen dagegen war es im Allgemeinen immer möglich, Kräfte zu finden, welche, nach ihnen wirkend, sich das Gleichgewicht halten, und es konnte daher eine ihrer Richtung und Intensität nach beliebig gegebene Kraft im Allgemeinen immer in 6 andere zerlegt werden, deren Richtungen beliebig gegeben sein konnten. Der Beisatz ,,im Allgemeinen" wurde durch die negative Bedingung bestimmt, dafs weder alle 6 gegebene Richtungen, noch einige derselben eine solche Lage gegegen einander hatten, bei welcher es möglich war, nach ihnen wirkende Kräfte zu finden, welche sich das Gleichgewicht halten.

Bei der Analogie, die zwischen Krïften und Drehungen statt findet, mufs daher auch, - um hier nur den letzterwähnten Fall zu berücksichtigen - jede Drehung, und somit jede Verrückung überhaupt, 6 andern Drehungen um eben so viele von einander unabhängige, sonst beliebig anzunehmende Axen gleich gesetzt werden können, so da/s, wenn ein Körper um sechs von einander unabhängige Axen drehbar ist, er auf jede mögliche Weise verrückt werden kann. Sechs oder weniger Axen werden wir aber von einander unabhängig nennen; wenn es nicht möglich ist, Drehungen um dieselben ausfindig zu machen, welche sich gegen einander aufheben; woraus zugleich erhellet, dafs 7 oder mehrere Axen nio von einander unabhängig sein können.

Zufolge des vorigen §. mufs sich demnach, wenn voh irgend einer Verrückung die Momente in Bezug auf 6 von einander unabhängige Axen gegeben sind, aus ihnen das Moment für jede Yte Axe herleiten lassen. Zerlegt man nämlich, wie es nach dem oben Bemerkten immer möglich ist, eine Drehung um die 7te Axe in 6 Drehungen um die 6 erstern, so 
wird die Summe der Producte aus jeder dieser 6 Drehungen in das auf die jedesmalige Drehungsaxe bezogene Moment der Verrückung gleich sein dem Producte aus der Drehung um die 7te Axe in das gesuchte Moment für dieselbe Axe.

\section{§. 16.}

Um auch diese Sätze durch ein Beispiel zu erlikutern, wollen wir eine Drehung $\rho$ um eine beliebig gegebene $A x e P Q$ in 6 Drehungen um die 6 Kanten einer dreiseitigen Pyramide $A B C D$ - oder, was auf dasselbe hinauskommt, eine nach $P Q$ gerichtete Kraft $\rho$ in 6 andere nach den Kanten von $A B C D$ gerichtete - zu zerlegen suchen. Dieses mufs möglich sein, da, wie man leicht wahrnimmt, von 6 Kräften, welche nach den 6 Kanten einer Pyramide wirken, keine die Resultante von den 5 übrigen oder von einigen derselben sein kann.

Zur Lösung dieser Aufgabe mag die in \$. 102. meines Lehrbuchs angegebene, auf den Hauptsatz in der Theorie der Momente gegründete Methode dienen, - Seien die gesuchten Drehungen um die Axen

$$
\begin{aligned}
& A D, B D, C D, B C, C A, A B \\
& \text { resp. }=\alpha, \quad \beta, \quad \gamma_{2} \quad \delta, \quad \varepsilon, \quad \zeta .
\end{aligned}
$$

Indem wir nun die Momente sämmtlicher Drehungen auf eine beliebig zu wählende $A \times e X Y$ beziehen, ist das Moment einer, sowohl hinsichtlich ibrer Axe, als ibrer Gröfse, durch $A D$ ausgedrückten Drehung $=$ der Pyramide $A D X Y$; folglich, wenn die Grölse nicht $A D$ sondern 1 ist, $=\frac{1}{A D} A D X Y$, und wenn $\alpha$ die Grölse der Drehung ausdrückt, $=\frac{a}{A D} A D X Y$. Eben so ist das Moment der Drehung $\beta$ um die Axe $\boldsymbol{B D}=\frac{\beta}{B D} \boldsymbol{B C X Y}$, u. s. w. Da nun die Drehungen $\left.\alpha, \beta, \ldots\right\}$ um die Axen $A D, B D, \ldots A B$ gleiche Wirkung mit der Drehung $\rho$ um die Axe $\boldsymbol{P Q}$ haben sollen, so ist nach dem Satze von den Momenten (\$.13.):

$$
\text { I. }\left\{\begin{array}{c}
\frac{\alpha}{A D} A D X Y+\frac{\beta}{B D} B D X Y+\frac{\gamma}{C D} C D X Y \\
+\frac{\delta}{B C} B C X Y+\frac{\varepsilon}{C A} C A X Y+\frac{\zeta}{A B} A B X Y
\end{array}\right\}=\frac{\rho}{P Q} P Q X Y \text {. }
$$

Man lasse jetzt die willkührlich zu nehmenden Puncte $X$ und $Y$ mit $A$ und $D$ zusammenfallen, so wird jede der 5 Pyramiden $A D X Y, B D X Y$, $C D X Y, C A X Y, A B X Y$ Null, und die Gleichung I. reducirt sich auf:

$$
\text { 1. } \frac{\delta}{B C} B C A D=\frac{\varrho}{P Q} P Q A D \text {. }
$$


Auf gleiche Weise findet sich, wenn man $X Y$ nach und nach mit $\boldsymbol{B D}, \boldsymbol{C D}, \boldsymbol{B C}, \boldsymbol{C A}, \boldsymbol{A B}$ coïncidiren lärst :

$$
\text { 2. } \frac{\varepsilon}{C A} C A B D=\frac{Q}{P Q} P Q B D \text {, }
$$

$$
\begin{aligned}
\text { 3. } \frac{\zeta}{A B} A B C D & =\frac{\varrho}{P Q} P Q C D, & \text { 4. } \frac{\alpha}{A D} \cdot A D B C & =\frac{\varrho}{P Q} P Q B C, \\
\text { 5. } \quad \frac{\beta}{B D} B D C A & =\frac{\varrho}{P Q} P Q C A, & \text { 6. } \frac{\gamma}{C D} C D A B & =\frac{\varrho}{P Q} P Q A B,
\end{aligned}
$$

Durch diese 6 Gleichungen werden aber die Gröfsen der Drehungen $\alpha, \beta, \gamma, \delta, \varepsilon, \zeta$ vollkommen bestimmt.

Sind nun von irgend einer Verrückung die Momente in Bezug auf die Axen

$$
\begin{array}{rcccccc}
A D, & B D, & C D, & B C, & C A, & A B, & P Q \\
\text { resp. }=a, & b, & c, & d, & e, & f, & r,
\end{array}
$$

so ist nach $\S .15 .:$

$$
a \alpha+b \beta+c \gamma+d \delta+e \varepsilon+f \zeta=r \rho,
$$

oder, wenn man für $\alpha, \beta, \ldots \zeta$ ihre Werthe au's (1),.... (6.) setzt und dabei berücksichtigt, dafs die Pyramiden $B C A D, C A B D, A B C D, \ldots$ nicht allein ihrem absoluten Werthe, sondern auch ihren Zeichen nach einander *) gleich sind:

$$
\text { II. }\left\{\begin{array}{r}
\text { a.AD.PQBC }+ \text { b. BD.PQCA + c.CD.PQAB } \\
+ \text { d. BC.PQAD }+ \text { e.CA.PQBD +f.AB.PQCD } \\
=r \cdot P Q \cdot A B C D
\end{array}\right.
$$

und dieses ist die Gleichung, mittelst welcher man, wenn die Figur $A B C D P Q$ und die Momente $a, b, \ldots f f$ einer Verrückung für die Kanten der Pyramide $A B C D$, als Axen, gegeben sind, das Moment $\boldsymbol{r}$ derselben Verrückung für $\boldsymbol{P Q}$ finden kann.

\section{\$. 17.}

Zusätze. a) Substituirt man in der Gleichung (I.) für $\alpha, \beta, \ldots$ $\ldots \zeta$ ihre Werthe aus (1.), ....(6.), so kommt

$$
\text { III. }\left\{\begin{array}{r}
\text { BCPQ.ADXY }+C A P Q \cdot B D X Y+A B P Q \cdot C D X Y \\
+A D P Q . B C X Y+B D P Q \cdot C A X Y+C D P Q \cdot A B X Y \\
=A B C D \cdot P Q X Y
\end{array}\right.
$$

eine Relation zwischen 14 Pyramiden bei einem beliebig angenommenen Systeme von 8 Puncten im Raume, deren Symmetrie hinsichtlich $\boldsymbol{A B C D}$, $P Q, X Y$ in die Augen springt.

*) Lehrb. d. St. §. 63.1. 
13. A. E. Möbius, über die Zusammensetzung unendlich kleiner Drelungen. 209

b) Läfst man $X Y$ mit $P Q$ zusammenfallen, so reducirt sich III. auf

IV. $\mathrm{BCPQ} \cdot A D P Q+C A P Q \cdot B D P Q+A B P Q \cdot C D P Q=0$ : eine Relation zwischen 6 Pyramiden bei 8 Puncten im Raume ${ }^{*}$ ).

c) Setzt man in IV. für die 6 Pyramiden ihre aus (1.).... (6.) fliefsenden Werthe, so erhält man

$$
\text { V. } \frac{\alpha}{A D} \cdot \frac{\delta}{B C}+\frac{\beta}{B D} \cdot \frac{\varepsilon}{C A}+\frac{\gamma}{C D} \cdot \frac{\zeta}{A B}=0 \text {, }
$$

welches die Relation ist, die zwischen 6 Drehungen $\alpha, \ldots\}$ um die Kanten $A D, \ldots A B$ einer Pyramide Statt finden müssen, wenn sich die Drehungen auf eine einzige sollen reduciren lassen; - also auch die Bedingungsgleichung, wenn 6 nach den Kanten einer Pyramide gerichteten Kräfte $\alpha, \ldots . \zeta$ mit einer einzigen Kraft gleiche Wirkung haben sollen.

\section{§. 18.}

Der Satz, dafs ein Körper vollkommen frei beweglich ist, wenn er um 6 von einander unabhängige Axen gedreht werden kann, gehört unstreitig zu den merkwürdigsten in der Theorie der Drehungen. Ein Fall, in welchem man sich von diesem Satze sehr leicht ohne alle Rechnung überzeugen kann, ist der, wenn zu den 6 Axen die 6 Kanten $D A, D B, D C$,

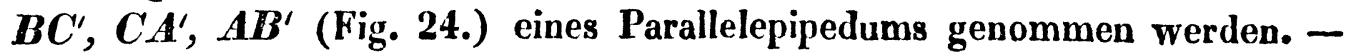
$A, B, C$ sind bei demselben die der Ecke $D$ zunächst liegenden Ecken, und $A^{\prime}, B^{\prime}, C^{\prime}, D^{\prime}$ liegen den $A, B, C, D$ diagonal gegenüber.

Jede Verrückung kann nämlich in eine Drehung um einen beliebig zu nehmenden Punct $D$ und in eine parallele Fortrückung aufgelöset werden. Hiervon läfst sich die Drehung um $D$ in drei andere $\alpha, \beta, \gamma$ um drei durch $D$ gehende $\operatorname{Axen} D A, D B, D C$ zerlegen, und eben so kann man die parallele Fortrückung in drei andere zerlegen, welche hier auf den Ebenen $B C, C A, A B$ perpendicular seien. Für die auf $B C$ perpendiculare Fortrückung aber können zwei Drehungen $\beta^{\prime},-\beta^{\prime}$ um zwei parallele Axen $\boldsymbol{C A}^{\prime}, \boldsymbol{D B}$ dieser Ebene gesetzt werden. Verwandelt man nun eben so die auf $\boldsymbol{C A}$ und $\boldsymbol{A B}$ perpendicularen Fortrückungen in die Drehungen $\gamma^{\prime},-\gamma^{\prime}$ um $\boldsymbol{A B}, \boldsymbol{D C}$ und in die Drehungen $\alpha^{\prime},-\alpha^{\prime}$ um $B C^{\prime}, D A$, so wird die ganze Verrückung reducirt auf die 6 Drehungen um die Axen

$$
\alpha-\alpha^{\prime}, \beta-\beta^{\prime}, \gamma-\gamma^{\prime}, \quad \alpha^{\prime}, \quad \beta^{\prime}, \quad \gamma^{\prime}
$$

$$
D A, \quad D B, \quad D C, \quad B C^{\prime}, C A^{\prime}, A B^{\prime} \text {. }
$$

Letztere Relation fudet sich bereits in des Verf. „Baryc. Calcul \$. 170." gegeben. 
Zur Bestimmung der Werthe von $\alpha, \alpha^{\prime}, \beta, \ldots$, wenn irgend eine Drehung $\rho$ um eine $A x e P Q$ gegeben ist, die in Drehungen um die 6 Axen $D A, D B, \ldots$ zerlegt werden soll, kann man wiederum die vorhin erläuterte Methode mit Vortheil anwenden. Man schreibe für

$D A, D B, D C, B C^{\prime}, C A^{\prime}, A B^{\prime}, P Q$ der Kürze wegen

$$
a, \quad b, \quad c, \quad a^{\prime}, b^{\prime}, c^{\prime}, \quad r \text {, }
$$

und verstehe eben so unter $a b^{\prime}$, ar, .... die Pyramiden DACA ${ }^{\prime}$, DAPQ, ... Alsdann ist, wie in §.16., wenn man die Momente sämmtlicher Drehungen auf die unbestimmte Axe $x$ bezieht:

$$
\frac{\alpha-\alpha^{\prime}}{a} a x+\frac{\beta-\beta^{\prime}}{b} b x+\frac{\gamma-\gamma^{\prime}}{c} c x+\frac{\alpha^{\prime}}{a} a^{\prime} x^{*}+\frac{\beta^{\prime}}{b^{\prime}} b^{\prime} x^{\star}+\frac{\gamma^{\prime}}{c^{\prime}} c^{\prime} x=\frac{\rho}{r} r x^{*} \text {. }
$$

Diese Gleichung reducirt sich, wenn man $x$ zuerst mit $a$ und dann mit $a^{\prime}$ zusammenfallen lärst, auf

$$
\frac{\beta^{\prime}}{b^{\prime}} b^{\prime} a=\frac{\varrho}{r} r a \text { und } \frac{\gamma-\gamma^{\prime}}{c} c a^{\prime}+\frac{\beta^{\prime}}{b^{\prime}} b^{\prime} a^{\prime}+\frac{\gamma^{\prime}}{c^{\prime}} c^{\prime} a^{\prime}=\frac{\varrho}{r} r a^{\prime} \text {. }
$$

Man gewahrt aber leicht, dafs die Pyramiden $b^{\prime} a, c a^{\prime}, b^{\prime} a^{\prime}$ und $c^{\prime} a^{\prime}$ sowohl dem Zeichen, als dem absoluten Werthe nach einander gleich sind, indem jede von ihnen dem 6 ten Theile des Parallelepipedums $D D^{\prime}$ gleich ist. Setzt man daher $\frac{e}{r} \cdot \frac{1}{b^{\prime} a}=R$ und bemerkt, dafs $c^{\prime}=c$, so ziehen sich die zwei erhaltenen Gleichungen zusammen in

und eben so folgt:

$$
\frac{\beta^{\prime}}{b^{\prime}}=R . r a \text { und } \frac{\gamma}{c}+\frac{\beta^{\prime}}{b^{\prime}}=R \cdot r a^{\prime},
$$

$$
\begin{array}{ll}
\frac{\gamma^{\prime}}{c^{\prime}}=R . r b, & \frac{\alpha}{a}+\frac{\gamma^{\prime}}{c}=R . r b^{\prime}, \\
\frac{\alpha^{\prime}}{a^{\prime}}=R . r c, & \frac{\beta}{b}+\frac{\alpha^{\prime}}{a^{\prime}}=R . r c^{\prime},
\end{array}
$$

wenn man $x$ der Reihe nach mit $b, b^{\prime}, c, c^{\prime}$ identisch werden lälst. Mittelst dieser Gleichungen aber werden die 6 Drehungen $\alpha, \ldots . \gamma^{\prime}$ durch die Drehung $\rho$ und durch die Lage der Axe der letztern gegen die Axen der erstern bestimmt; wie verlangt wurde.

Läfst man in der Hauptgleichung $x$ noch mit $r$ zusammenfallen und substituirt dann für $a r, b r, \ldots . c^{\prime} r$ ihre durch die 6 letzten Gleichungen bestimmten Werthe, so ergiebt sich, nach leichter Reduction:

$$
\frac{\alpha}{a} \cdot \frac{\beta^{\prime}}{b^{\prime}}+\frac{\beta}{b} \cdot \frac{\gamma^{\prime}}{c^{\prime}}+\frac{\gamma}{c} \cdot \frac{\alpha^{\prime}}{a^{\prime}}=0,
$$

und dies ist die Bedingung, unter welcher sich im vorliegenden Falle die 6 Drehungen $\alpha, \ldots \gamma^{\prime}$ auf eine einzige reduciren lassen. 
In dem Bisherigen wurden die Axen, um welche ein Körper nach und nach gedreht wird, als unbeweglich betrachtet. Indessen ist dieses nicht durchaus nothwendig. Denn unter der hier immer geltenden Voraussetzung, dafs jeder Drehungswinkel unendlich klein sei, sind zwei gleiche Drehungen a um zwei ihrer Lage nach unendlich wenig verschiedene Axen $a$ und $a^{\prime}$ (d. h. um zwei Axen, deren kürzester Abstand von einander und deren gegenseitige Neigung unendlich klein sind) als gleichwirkend anzusehen, indem, wenn ein Punct $A$ durch die Drehung $\alpha$ um $a$ von $A$ nach $\boldsymbol{B}$ und durch die Drehung $\alpha$ um $a^{\prime}$ von $\boldsymbol{A}$ nach $B^{\prime}$ gebracht wird, $\boldsymbol{B B}^{\prime}$ eine unendlich kleine Linie der zweiten Ordnung ist, während $A B$ und $\boldsymbol{A B}^{\prime}$ von der ersten Ordnung sind. Die Gesammtwirkung von mehrern Drehungen wird folglich noch dieselbe sein, wenn wir die Drebungs-Axen mit dem beweglichen Körper selbst fest verbunden annehmen; als wodurch es geschieht, dafs bei jeder Drehung die Axen aller jedesmal übrigen Drehungen um ein unendlich Weniges mit verstellt werden.

\section{§. 20.}

Der Umstand, dafs eine unendlich kleine Veränderung der Lage der Axen auf die Lage des um sie gedrehten Körpers keinen Einflufs hat, kann uns veranlassen, unsere Betrachtung noch weiter auszudehnen und mit einem Systeme von 6 Axen z. B., welche der Reihe nach $a, b, c, d, e, f$ heilsen, 7 Körper $A, B, \ldots F, G$ dergestalt fest verbunden anzunehmen, dafs $A$ und $B$ um die gemeinschaftliche Axe $a ; B$ und $C$ um die gemeinschaftliche Axe $b$ und $C$ und $D$ um $c$ u. s. w. gedreht werden können. Bleibe nun $A$, folglich auch $a$, in Ruhe, und werde $B$ mit den übrigen Körpern $C, D, \ldots G$, als ein festes Ganzes um $a$, um den Winkel $\alpha$ gedreht. Man drehe hierauf, während $A$ und $B$, mithin auch $a$ und $b$ in Ruhe bleiben, den Körper $C$, in ungë̈nderter Verbindung mit den übrigen Körpern $D, \ldots, G$, um $b$ um den Winkel $\beta$, u. s. w., und endlich den Körper $G$ allein um $f$ um den Winkel $\zeta$, während alle vorhergehenden Körper $A, \ldots F$ unbewegt bleiben. Nach allen diesen Drehungen wird der Körper $G$ seine Lage eben so geändert haben, als wenn er nach und nach um die festen $A x e n a, b, \ldots f$ resp. um die Winkel $\alpha, \beta, \ldots\}$ gedreht worden wäre. Da nun durch die eben beschriebenen 6 Drehungen das System der 6 Körper $B, C, \ldots G$ in jede von der anfänglichen unendlich wenig verschiedene Lage, die es, 
dem Zusammenbange der Körper durch die Axen gemärs, anzunehmen vermag, gebracht werden kans, und da ein um 6 von einander unabhingige Axen drehbarer Körper jeder Verrückung fähig ist, so schliefsen wir:

Wenn von melirern Körpern, in einer gewissen Ordnung genommen, je zwei nüchstfolgende um eine gemeinschuftliche Axe drehbar sind, so hat, wenn der erste festgehalten wird, erst der siebente eine vollkommen freie Beryeglichkieit, und dieses auch nur dann, wenn die sechs, diese sieben Körper verbindenden Axen in eine von einander unabhüngige Lage gebracht werden können.

Als einfaches Beispiel hierzu kann Fig. 25. dienen, wo von 7 Quadraten $1,2,3,4,5,6,7$ je zwei nächstfolgende eine Seite gemein haben. Denkt man sich diese Seite als Axe, um welche die zwei durch sio verbundenen Quadrate drehbar sind, 80 kann gegen das Iste Quadrat erst das 7 te in jede beliebige Lage - allerdings nur innerhalb gewisser Grenzen - gebracht werden. Die hierzu erforderliche Unabhängigkeit der Axen ergiebt sich daraus, dafs man mit den 7 Quadraten in ihrer Verbindung einen Würfel überdecken kann, bei welchem die, zwei nächstfolgenden Quadraten gemeinschaftlichen Seiten $A D, D B, B A^{\prime}, A^{\prime} D^{\prime}, D^{\prime} B^{\prime}, B^{\prime} A$ eine solche Lage gegen einander haben, dafs zwischen Krïften, welche diese 6 Seiten zu ihren Richtungen haben, kein Gleichgewicht möglich ist. - Sehr leicht kann man sich von der vollkommenen gegenseitigen Beweglichkeit der beiden äufsersten Quadrate auch durch Proben überzeugen, wenn man Fig. 25. etwa in steifem Papier ausschneidet und dasselbe nach den Seiten bricht, in denen die Quadrate aneinander grenzen.

Schliefslich noch die Bemerkung, dafs uns der eben erläuterte Satz zum Aufschlufs über eine von der Natur bei den Gliedmafsen der Krebse (und wahrscheinlich auch anderer Insecten) getroffene. Einrichtung dienen kann. Bei diesen Thieren bestehen nämlich die Beine, so wie auch die Gliedmafsen, an deren Enden die Scheeren sitzen, aus sechs Gliedern, die unter sich und mit dem Körper selbst durch sechs Axengelenke verbunden $\operatorname{sind}^{*}$ ). Hierdurch aber wird nach dem Vorigen der Z $\mathbf{Z}$ weck erreicht, dafs, während der Körper ruht, das äufserste Glied jedes Beines vollkommen freie Beweglichkeit hat.

*) Observationes de scoleto astaci fluriatilis et marini, dissertatio inauguralis auctore C. E. Hasse. Lipsiac. 1823. Pag. 27. 